\title{
The XY Scanner - A Versatile Method of the Absolute End-to-End Calibration of Fluorescence Detectors
}

\author{
Christoph M. Schäfer ${ }^{a, *}$ on behalf of the Pierre Auger ${ }^{b}$ Collaboration \\ (a complete list of authors can be found at the end of the proceedings) \\ ${ }^{a}$ Institute for Astroparticle Physics, Karlsruhe Institute of Technology $\mathbf{\mathbf { K }} \mathbf{\mathbf { T }}$, Germany \\ ${ }^{b}$ Observatorio Pierre Auger, Av. San Martín Norte 304, 5613 Malargüe, Argentina \\ E-mail: spokespersons@auger.org
}

\begin{abstract}
One of the crucial detector systems of the Pierre Auger Observatory is the fluorescence detector composed of 27 large-aperture wide-angle Schmidt telescopes. In the past, these telescopes were absolutely calibrated by illuminating the whole aperture with a uniform large-diameter light source. This absolute calibration was performed roughly once every three years, while a relative calibration was performed on a nightly basis. In this contribution, a new technique for an absolute end-to-end calibration of the fluorescence telescopes is presented. For this technique, a portable, calibrated light source mounted on a rail system is moved across the aperture of each telescope instead of illuminating the whole aperture at once. A dedicated setup using a combination of NIST traceable photodiodes to measure the mean intensity and a PMT for pulse-to-pulse stability tracking has been built for the absolute calibration of the light source. As a result of these complementary measurements, the pulse-to-pulse light source intensity can be known to the $3.5 \%$ uncertainty level. The analysis of the readout of the PMT camera at each position of the light source together with the knowledge of the light source emission provides an absolute end-to-end calibration of the telescope. We will give a brief overview of this novel calibration method and its current status as well as show preliminary results from the measurement campaigns performed so far.
\end{abstract}

$37^{\text {th }}$ International Cosmic Ray Conference (ICRC 2021)

July 12th - 23rd, 2021

Online - Berlin, Germany

\footnotetext{
${ }^{*}$ Presenter
} 

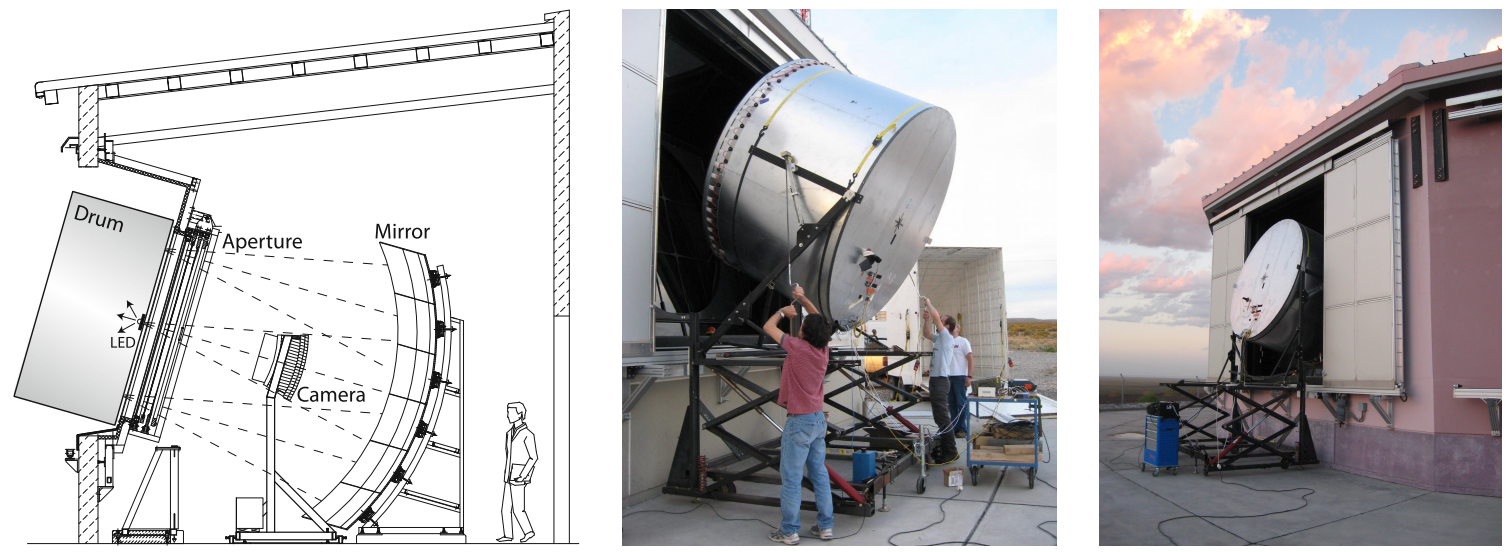

Figure 1: Schematics (left) and pictures of the installation (middle) and the measurement position (right) of the large-diameter calibration light-source (drum). Schematics is taken from Ref. [2].

\section{Introduction}

The Pierre Auger Observatory [1] consists of a surface-detector array (SD) employing 1660 water-Cherenkov detector stations distributed across an area of $\sim 3000 \mathrm{~km}^{2}$ and overlooked by 27 large telescopes located at four different sites, forming the fluorescence detector (FD). In particular FD and SD systems are used to observe cosmic-ray induced air-showers by a hybrid approach. The large SD array samples particles reaching the ground, measuring a footprint of the air shower. Additionally the FD detects the fluorescence light which is emitted by the air above the ground array upon passage of charged shower particles. The detection of the shower-induced fluorescence light is then used to reconstruct the longitudinal profile of the shower. The total energy deposited by the air shower is proportional to the energy of the primary cosmic ray and the integral of this longitudinal profile. For this purpose, the exact conversion of a measured signal by the FD camera to an incident photon flux at the aperture window has to be known.

The FD telescopes of the Pierre Auger Observatory consist of a 440 pixel PMT-camera which is located at the focal plane of a large spherical mirror. Each $100 \mu$ s pixels are read out via a flash analog-to-digital converter. The aperture window has a diameter of $\sim 2.2 \mathrm{~m}$ and is covered by a filter which is transparent only to UV photons with wavelengths between $\sim 290$ and $\sim 410 \mathrm{~nm}$. In addition, the filter protects the interior parts of the telescopes from wind, dust, and rain. Behind the filter, a corrector lens is installed, which partially corrects for the spherical aberration of the mirror. To reduce weight, cost, and construction effort, the corrector lens is build as a ring covering only the outer $\sim 25 \mathrm{~cm}$ of the aperture.

For an absolute end-to-end calibration of the FD telescope the entire aperture has to be illuminated with a known photon flux and all pixels of the camera are read out simultaneously. The known photon flux together with the signal captured by each PMT provides a calibration constant for each individual PMT. These calibration constants include all effects induced by optical components of the telescope, like e.g. the transmittance of the filter, reflectivity of the mirror, multiple reflections within the telescope, and also various PMT effects such as e.g. quantum and collection efficiency, amplification gains, and the digital conversion factors.

In the past the absolute calibration of the FD telescopes was performed with a $2.5 \mathrm{~m}$ diameter 


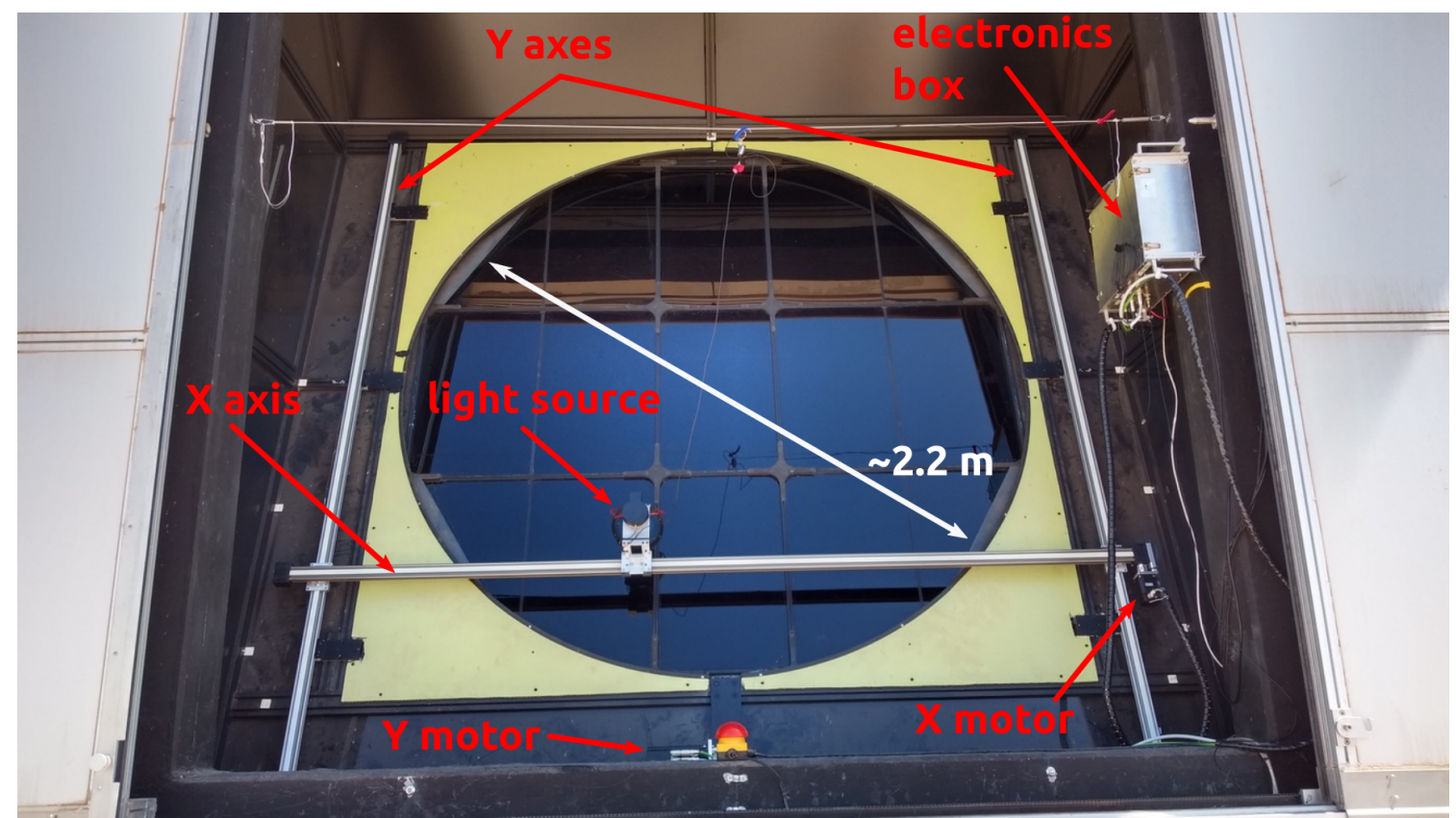

Figure 2: Photograph of a complete XY-scanner system installed onto an aperture of a FD telescope. All important components are labeled.

uniform light source which covered the entire aperture of the telescope [3, 4]. This light source, called drum because of its shape and appearance, was directly mounted on top of the UV filter of the FD telescope during periodic calibration campaigns. Drawing and photos of the installation of the drum are shown in Fig. 1. Because of its sheer size, handling, installing, and operating the drum is rather laborious and for this task requires help of several persons. The rather large amount of personpower needed to perform the absolute FD calibration with the drum was one of the main reasons why the calibration of the FD telescopes was performed rather infrequently, only roughly once every three years. However, a relative calibration, which is executed at the beginning and end of every data-taking night, tracks the absolute calibration over time and thus provides a reliable calibration of each telescope for the nights between the drum absolute-calibration campaigns. The results from the latest drum calibration-campaign show that the relative calibration system delivers excellent tracking ability. In fact, the difference between the tracked calibration constants and the absolute calibration constants obtained during that latest drum calibration-campaign was on average less than $1 \%$.

\section{The XY Scanner}

In the following a novel technique of performing the absolute calibration of the FD telescopes is introduced and discussed. This new absolute calibration system employs a portable light source which is much smaller than the drum introduced above. Its small size enables us to transport the source easily between (a) the FD telescopes and sites and between (b) the Observatory and calibration laboratories on other continents to perform precise measurements of the source properties and its characteristics. More details on the light source are given in Section 3. 
As a consequence of the small physical size of the light source, its output aperture window is much smaller than the aperture of the FD telescopes. To overcome this difference in sizes we move the light source across the aperture window, mimicking the illumination of the entire aperture as was the case in the drum calibration.

The system which moves the light source in front of the aperture is called the XY-Scanner. It consists of three motorized linear stages, two of them are mounted vertically as Y-axes while the other is mounted horizontally as X-axis. The two Y-axes are installed permanently in the aperture box, left and right of the aperture opening while both ends of the $\mathrm{X}$-axis are mounted onto the sliding carriages of the Y-axes. The horizontal X-axis is only installed during calibration measurements done with the XY-Scanner, otherwise it is stored inside the FD telescope building. For their protection the Y-axes are covered by a metal shielding during most of the time when no calibration measurements are performed. We decided to employ the drylin ${ }^{\circledR} \mathrm{W}$ profiles produced by the company igus [5] as linear stages for all axes. These components are designed to be maintenancefree and very insensitive to influences such as dirt, water or heat. These characteristics make the drylin ${ }^{\circledR}$ components a almost ideal fit for the rough environment of the Argentinian pampa. A photograph of a complete XY-Scanner system installed at a FD telescope is shown in Fig. 2. To evade synchronization between the motors operating the two vertical Y-axes, both Y-axes are driven by a single motor located at the lower center of the aperture box. The Y-axes and the motor are mechanically connected via a shaft. The motor for the X-axis is directly attached to its right end. Both axes are equipped with a braking system which is enabled during actual data-taking, i. e., once the light source moved to its desired position both motors are switched off and the brake system holds everything in position. With this procedure, possible electromagnetic interference from current flows within the motors are reduced to a minimum. The whole positioning system has a relative precision in the sub-millimeter range and automatically corrects for possible lost steps during all movements.

Status at the Pierre Auger Observatory So far we have installed $10 \mathrm{XY}$-Scanner system at three different FD sites and we plan to equip all 27 FD telescopes of the Pierre Auger Observatory with an XY-Scanner system in the foreseeable future. In fact, the material needed for the installation for the remaining 17 system is already stored at the central campus in Malarguie and will be probably installed in late 2021 or 2022, depending on the situation with the global pandemic. Once all 27 FD telescopes will be equipped with a XY-Scanner system, we aim to have regular calibration campaigns and estimate the absolute end-to-end calibration on a yearly basis.

\section{The Calibration Light-Source}

As light source for the XY-Scanner we employ a $\sim 13.5 \mathrm{~cm}(5.3 ")$ diameter general purpose integrating sphere of the type 3P-GPS-053-SL manufactured by labsphere [6]. The use of a general purpose integrating sphere as an extended uniform light source is summarized in [7]. This integrating sphere has one large exit port of $\sim 6.35 \mathrm{~cm}\left(2.5^{\prime \prime}\right)$ diameter which is reduced to $\sim 5.04 \mathrm{~cm}$ (2") by a port reducer. In addition there are two smaller $\sim 2.54 \mathrm{~cm}(1 ")$ diameter ports positioned in a plane parallel to the exit port. For the time of XY-Scanner calibration measurements at a FD telescope, the exit port faces towards the aperture window of that telescope while the two smaller 

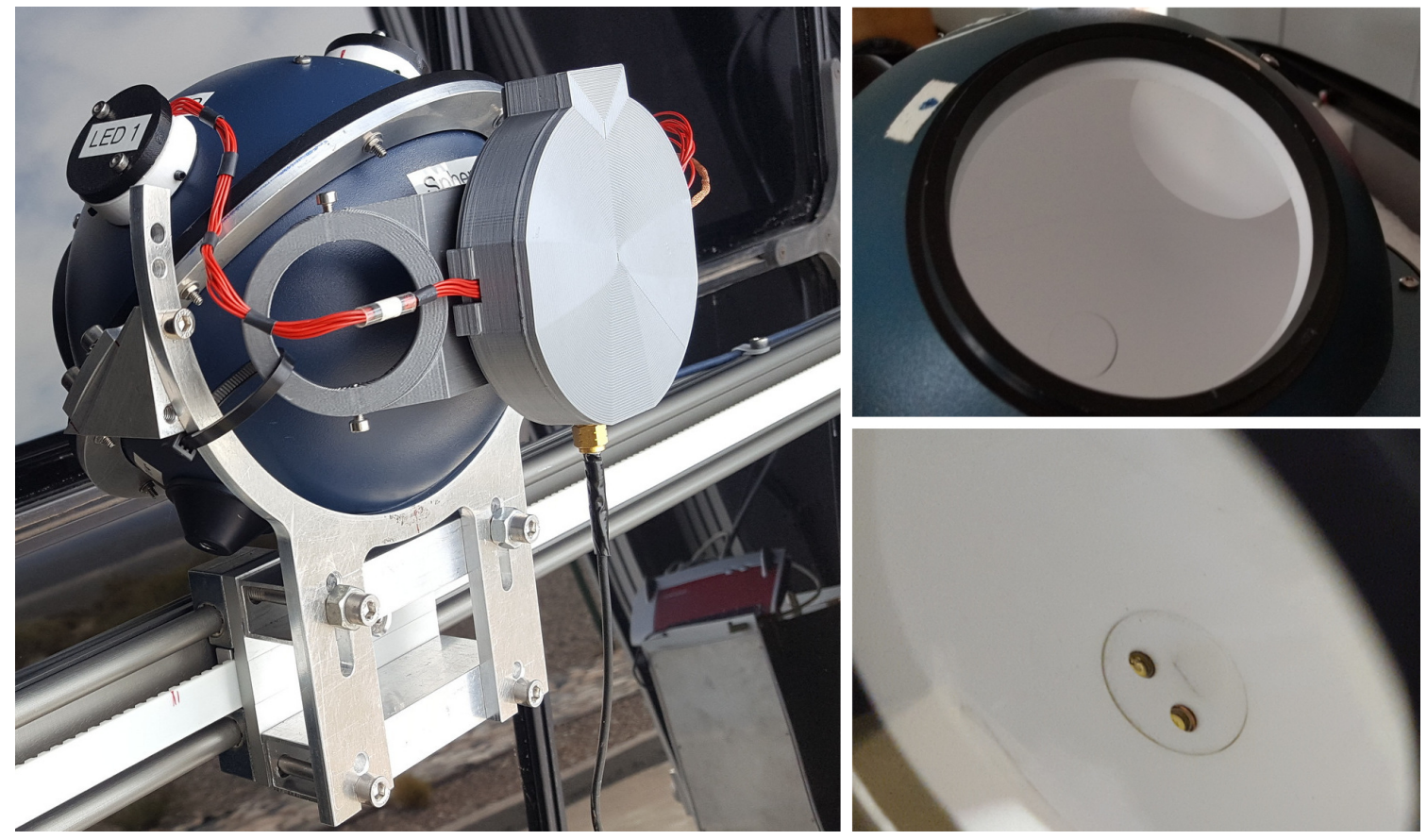

Figure 3: Left: Photograph of the integrating-sphere light-source mounted in front of one FD aperture at a XY-Scanner system. Right: Photos of the two baffles. The upper photo shows the original baffle covering the entire LED head. A photo of the modified baffle which only covers one of three LEDs is printed below.

ports are equipped with so called heads. In Fig. 3-left a photograph of the integrating-sphere lightsource installed at a XY-Scanner system is shown. One of theses heads holds a Hamamatsu S133644BQ photo-diode (PHD) which monitors the pulse-to-pulse stability of the light source itself, but is not considered for any absolute calibrations. The other head carries three light-emitting-diodes (LEDs), emitting at a wavelength of $\lambda \approx 365 \mathrm{~nm}$ of the type UVLED365-10E made by Roithner Lasertechnik [8]. These three LEDs mounted in that head are not operated simultaneously as each of them has its own purpose. The first LED is used for laboratory test measurements, the second one is used to track ageing effects of the other LEDs as well as the interior material of the integrating sphere itself, and the third LED is the actual light source used for calibration measurements. Both, the PHD and all three LEDs, are mounted into temperature stabilized copper disks which are heated to and stabilized at $30^{\circ} \mathrm{C}$. We chose this temperature setting to be well above the excepted ambient air temperature during nights at the FD sites in the Argentina. Therefore, it is sufficient to provide the heads with heating only to stabilize them at $30^{\circ} \mathrm{C}$ and no cooling is required, which would make the design of the head much more complicated. The readout of the PHD, monitoring and adjusting the temperatures, as well as operating the LEDs is performed by a micro controller of the MSP430 family. The micro controller, together with all other required components is installed onto a printed circuit board which is mounted outside the integrating sphere. The gray box on the back of the sphere in Fig. 3-left contains the micro controller and the circuit board. A single coaxial cable provides power to the board and in addition serves as trigger input line. Setting up the parameters of the light source and transferring data is realized via a wireless connection.

In the original general purpose version of the integrating sphere (as manufactured by labsphere) 

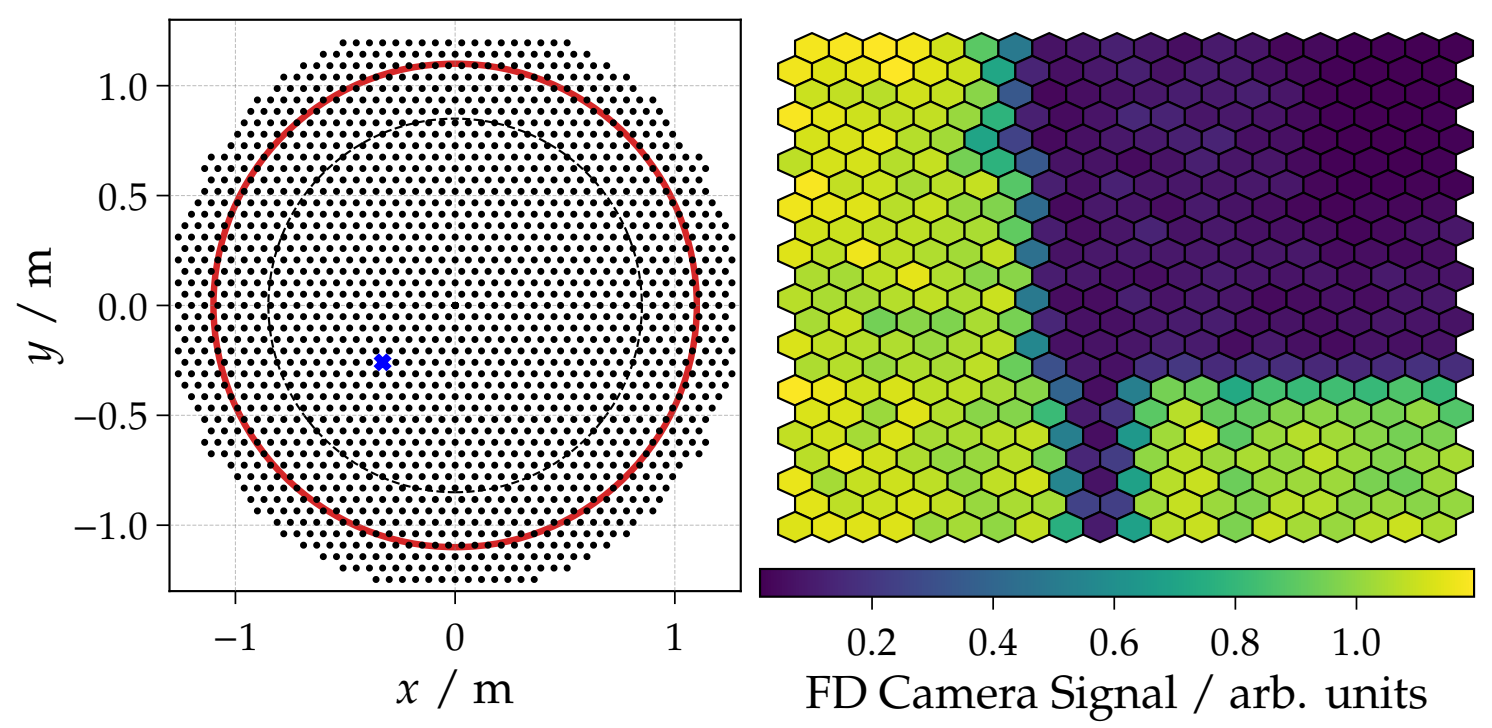

Figure 4: Left: All positions reached by the light source in front of the aperture of a FD telescope during a XY-Scanner measurement run are plotted as black dots. The red and black-dashed circles represent the edges of the aperture window and the corrector ring respectively. Right: Readout of the entire FD camera for the light source being at a certain position marked in blue on the plot on the left.

there is a so called baffle installed inside which prevents the direct light, emitted from the LEDs, to leave the sphere without any internal reflections. This baffle is a rather large shield located next to the port into which the LED head is plugged in. As a negative side effect this baffle influences the emission profile of the light source and introduces inhomogeneities. To reduce this unwanted influence of the baffle we reduced its size to a minimum so that it therefore shields only the direct emission of one of the three LEDs. The photographs on the right side of Fig. 3 show the unmodified baffle covering all three LEDs in the top photograph. The optimized, smaller baffle which covers only one of the three LEDs is displayed in the lower photograph. Size and position of the optimized baffle were estimated via simulations of the integrating sphere. In addition, to further improve the homogeneity of emission profile, a thin layer of a diffuse transmitting material (opal glass) was installed to cover this LED in an optimized version of the integrating sphere. With this modifications a nearly ideal Lambertian emission of the sphere is achieved.

The absolute calibration of the light source is performed in a dedicated setup built in the laboratory. This setup employs a combination of NIST traceable PHD and a PMT. The PMT traces the pulse-to-pulse stability of the emitted light flashes, while the PHDs measure the mean intensity of these pulses. With this complementary measurements, the absolute photon flux emitted by the light source is estimated to a $3.5 \%$ uncertainty level.

\section{The Measurement Procedure}

Measurements with the XY-Scanner are performed in a way that the light source is moved to uniformly distributed positions across the entire aperture window of a FD telescope. At each of the positions the light source emits a $5 \mu$ s long light pulse of fixed intensity and all 440 PMTs of the FD 

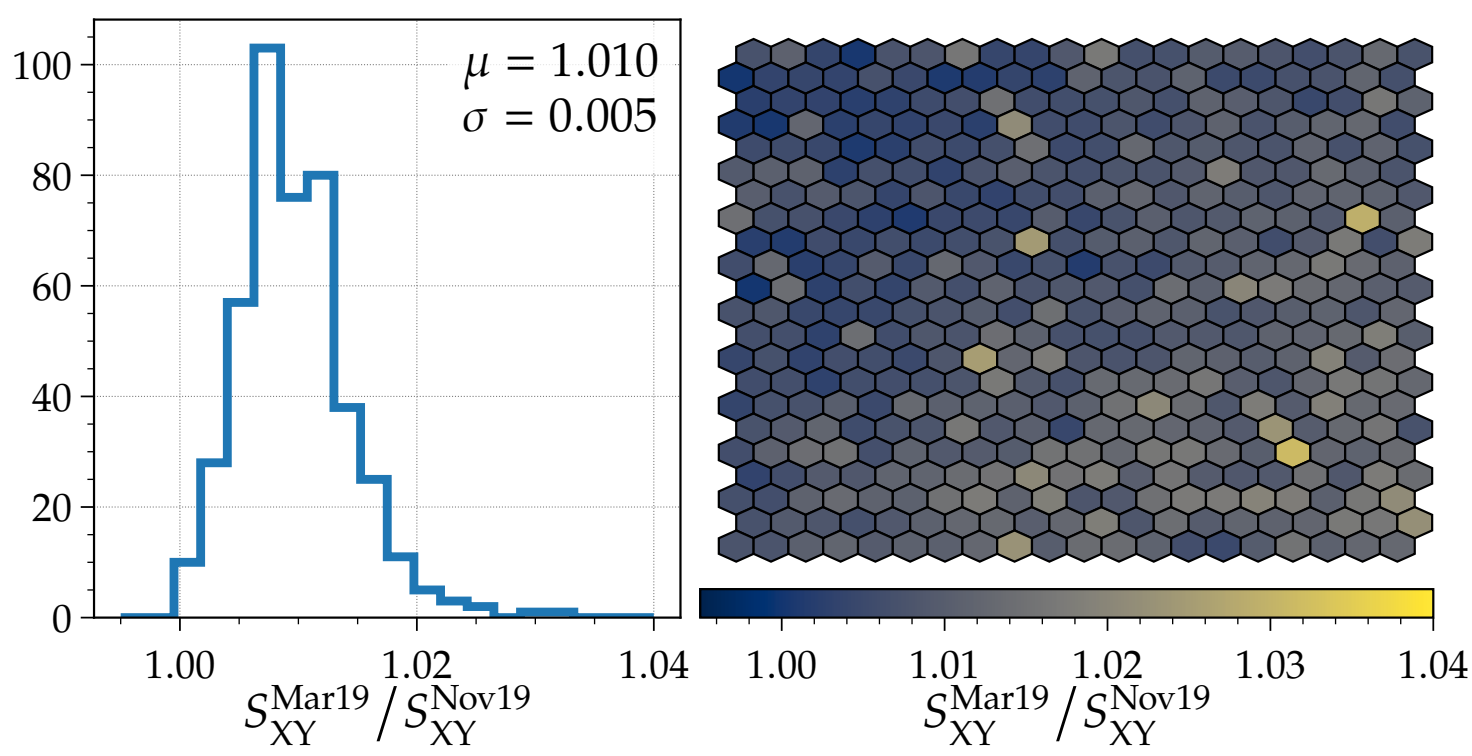

Figure 5: Left: Distribution the ratios of the captured signals by the FD PMTs between measurements performed in March and November 2019. Right: 2D pixel matrix of the ratios for the 440 individual PMTs of the camera.

camera are read out simultaneously. Reading out the whole camera is rather slow and thus limits the flashing frequency of the light source to $\sim 1 \mathrm{~Hz}$. Integrating over all flashes from all positions gives a similar illumination of the aperture window as the drum, as long as the positions are not too sparse. For an optimal coverage of the aperture window the measuring positions are distributed on a triangular grid. The distance between neighboring positions was determined on one hand to give a high coverage of the aperture opening and on the other hand to give a reasonable measuring time. For now we choose $6 \mathrm{~cm}$ as an optimal step size for the grid, which results in a suitable measuring time of less than one hour and gives a rather high aperture coverage of $\sim 65 \%$ for a integrating sphere with a $5.08 \mathrm{~cm}$ exit port diameter. The resulting grid contains $\sim 1700$ positions, which are shown as black dots on the left side of Fig. 4. The two concentric circles in this plot illustrate the edges of the aperture opening (red) and the corrector ring (black-dashed). For the position marked with a blue cross on this plot, the captured signal of the entire camera is shown on the right side of that Fig. 4 as a 2D pixel matrix. In this illustration a part of the shadow of the camera itself is visible as a dark rectangle in the upper right corner of the plot. In addition parts of the holding structure of the camera appear as a vertical line in the lower center.

Dedicated calibration measurements with the aim of estimating the absolute calibration of a FD telescope are performed only during nights which fulfill the standard requirements for ordinary data-taking of the FD.

The calibration of the FD telescopes is only reliable if the procedure is stable in time and thus provides reproducible results. To confirm the reproducibility of the XY scanner results, we performed measurements with the identical settings and setup during various calibration campaigns. The comparison of the captured signal in the FD PMTs during theses measurements then provide an estimation of the reproducibility of such measurements. In Fig. 5 the ratios between measurements 
performed at an interval of $\sim 9$ months are presented. Both plots of Fig. 5 are based on the same underlying data, namely they display both the ratios of the signal captured in the FD PMTs during XY scanner measurement in March 2019 and November 2019, but in two different representations. The left side of that Fig. 5 displays the distribution of the ratios, while the right side illustrates the signal ratio of individual PMTs as pixel matrix. On average the signals captured by each PMT differ by $\sim 1 \%$ and therefore we conclude the reproducibility of the XY scanner procedure on timescale of several months.

\section{Conclusion and Outlook}

In this proceeding, we presented a novel method of the absolute end-to-end calibration for FD telescopes, the XY-Scanner. The introduced technique employs a $\sim 13.5 \mathrm{~cm}(5.3 ")$ diameter general purpose integrating sphere as light source, which is moved across the aperture window by a motorized rail system consisting of two vertical axes and one horizontal axis. We made modifications to the integrating sphere to improve its emission characteristics and to match its emission profile closer to that of a Lambertian emitter. The photon flux emitted by the light source can be estimated at a 3.5\% level in a dedicated setup build in the laboratory. In addition we showed that the measurements performed with the XY-Scanner technique are reproducible on time intervals of several months.

The versatile setup of the XY-Scanner allows the straightforward installation of basically any light source, as long as its weight does not exceed the maximum weight rating of the scanner of $\sim 2.5 \mathrm{~kg}$. For example, we are currently developing and testing another directional light source which can be employed to measure imaging properties of the FD telescopes, like the point-spread-function.

At the moment there are 10 FD telescopes equipped with an XY-Scanner system and we plan to install XY-Scanner systems on the remaining FD telescopes in the foreseeable future.

Acknowledgments We gratefully acknowledge the help and support of the administrative and technical staff of the Pierre Auger Observatory in Malargüe. The authors gratefully acknowledge the support of the Ministry of Education, Youth and Sport of the Czech Republic project No. CZ.02.1.01/0.0/0.0/17_049/0008422.

\section{References}

[1] A. Aab et al. [Pierre Auger Coll.], Nucl. Instrum. Meth. A 798 (2015) 172-213, [1502.01323].

[2] J. Abraham et al. [Pierre Auger Coll.], Nucl. Instrum. Meth. A 620 (2010) 227-251, [0907 . 4282].

[3] J.T. Brack et al., Astropart. Phys. 20 (2004) 653.

[4] J.T. Brack et al., JINST 8 (2013) P05014, [1305 . 1329].

[5] igus GmbH, Spicher Str. 1a, D-51147 Köln, Germany, www . igus . de.

[6] Labsphere Inc., 231 Shaker St., North Sutton, NH 03260, USA, www . labsphere. com.

[7] M. Vacula et al., Optik 242 (2021) 167169.

[8] Roithner Lasertechnik GmbH, Wiedner Hauptstraße 76, A-1040 Vienna, Austria, www . roithner-laser . com. 


\section{The Pierre Auger Collaboration}

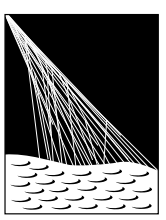

PIERRE

AUSGERVATORY

P. Abreu ${ }^{72}$, M. Aglietta ${ }^{54,52}$, J.M. Albury ${ }^{13}$, I. Allekotte ${ }^{1}$, A. Almela ${ }^{8,12}$, J. Alvarez-Muñiz ${ }^{79}$, R. Alves Batista ${ }^{80}$, G.A. Anastasi ${ }^{63,52}$, L. Anchordoqui ${ }^{87}$, B. Andrada ${ }^{8}$, S. Andringa ${ }^{72}$, C. $\mathrm{Aramo}^{50}$, P.R. Araújo Ferreira ${ }^{42}$, J. C. Arteaga Velázquez ${ }^{67}$, H. Asorey $^{8}$, P. Assis ${ }^{72}$, G. Avila ${ }^{11}$, A.M. Badescu ${ }^{75}$, A. Bakalova ${ }^{32}$, A. Balaceanu ${ }^{73}$, F. Barbato ${ }^{45,46}$, R.J. Barreira Luz $^{72}$, K.H. Becker ${ }^{38}$, J.A. Bellido ${ }^{13,69}$, C. Berat ${ }^{36}$, M.E. Bertaina ${ }^{63,52}$, X. Bertou ${ }^{1}$, P.L. Biermann ${ }^{b}$, V. Binet ${ }^{6}$, K. Bismark ${ }^{39,8}$, T. Bister ${ }^{42}$, J. Biteau ${ }^{37}$, J. Blazek ${ }^{32}$, C. Bleve ${ }^{36}$, M. Boháčová ${ }^{32}$, D. Boncioli ${ }^{57,46}$, C. Bonifazi ${ }^{9,26}$, L. Bonneau Arbeletche ${ }^{21}$, N. Borodai ${ }^{70}$, A.M. Botti ${ }^{8}$, J. Brack ${ }^{d}$, T. Bretz ${ }^{42}$, P.G. Brichetto Orchera ${ }^{8}$, F.L. Briechle ${ }^{42}$, P. Buchholz ${ }^{44}$, A. Bueno ${ }^{78}$, S. Buitink ${ }^{15}$, M. Buscemi ${ }^{47}$, M. Büsken ${ }^{39,8}$, K.S. Caballero-Mora ${ }^{66}$, L. Caccianiga ${ }^{59,49}$, F. Canfora ${ }^{80,81}$, I. Caracas ${ }^{38}$, J.M. Carceller ${ }^{78}$, R. Caruso ${ }^{58,47}$, A. Castellina ${ }^{54,52}$, F. Catalanii ${ }^{19}$, G. Cataldi ${ }^{48}$, L. Cazon ${ }^{72}$, M. Cerda ${ }^{10}$, J.A. Chinellato ${ }^{22}$, J. Chudoba ${ }^{32}$, L. Chytka ${ }^{33}$, R.W. Clay ${ }^{13}$, A.C. Cobos Ceruttii ${ }^{7}$, R. Colalillo ${ }^{60,50}$, A. Coleman ${ }^{93}$, M.R. Coluccia ${ }^{48}$, R. Conceição ${ }^{72}$, A. Condorelli ${ }^{45,46}$, G. Consolati ${ }^{49,55}$, F. Contreras ${ }^{11}$, F. Convenga ${ }^{56,48}$, D. Correia dos Santos $^{28}$, C.E. Covault ${ }^{85}$, S. Dasso ${ }^{5,3}$, K. Daumiller ${ }^{41}$, B.R. Dawson ${ }^{13}$, J.A. Day ${ }^{13}$, R.M. de Almeida ${ }^{28}$, J. de Jesús ${ }^{8,41}$, S.J. de Jong ${ }^{80,81}$, G. De Mauro ${ }^{80,81}$, J.R.T. de Mello Neto ${ }^{26,27}$, I. De Mitri ${ }^{45,46}$, J. de Oliveira ${ }^{18}$, D. de Oliveira Franco ${ }^{22}$, F. de Palma ${ }^{56,48}$, V. de Souza $^{20}$, E. De Vito ${ }^{56,48}$, M. del Río ${ }^{11}$, O. Deligny ${ }^{34}$, L. Deval ${ }^{41,8}$, A. di Matteo $^{52}$, C. Dobrigkeit ${ }^{22}$, J.C. D’Olivo ${ }^{68}$, L.M. Domingues Mendes ${ }^{72}$, R.C. dos Anjos ${ }^{25}$, D. dos Santos $^{28}$, M.T. Dova ${ }^{4}$, J. Ebr ${ }^{32}$, R. Engel ${ }^{39,41}$, I. Epicoco ${ }^{56,48}$, M. Erdmann ${ }^{42}$, C.O. Escobar ${ }^{a}$, A. Etchegoyen ${ }^{8,12}$, H. Falcke ${ }^{80,82,81}$, J. Farmer ${ }^{92}$, G. Farrar ${ }^{90}$, A.C. Fauth ${ }^{22}$, N. Fazzini ${ }^{a}$, F. Feldbusch ${ }^{40}$, F. Fenu ${ }^{54,52}$,

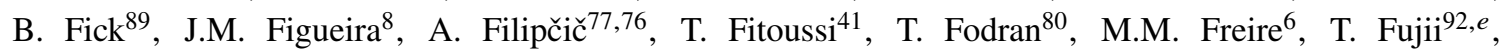
A. Fuster $^{8,12}$, C. Galea ${ }^{80}$, C. Galelli ${ }^{59,49}$, B. García ${ }^{7}$, A.L. Garcia Vegas ${ }^{42}$, H. Gemmeke ${ }^{40}$, F. Gesualdi ${ }^{8,41}$, A. Gherghel-Lascu ${ }^{73}$, P.L. Ghia ${ }^{34}$, U. Giaccari ${ }^{80}$, M. Giammarchi ${ }^{49}$, J. Glombitza ${ }^{42}$, F. Gobbi ${ }^{10}$, F. Gollan ${ }^{8}$, G. Golup ${ }^{1}$, M. Gómez Berisso ${ }^{1}$, P.F. Gómez Vitale ${ }^{11}$, J.P. Gongora ${ }^{11}$, J.M. González ${ }^{1}$, N. González ${ }^{14}$, I. Goos ${ }^{1,41}$, D. Góra ${ }^{70}$, A. Gorgi ${ }^{54,52}$, M. Gottowik ${ }^{38}$, T.D. Grubb ${ }^{13}$, F. Guarino ${ }^{60,50}$, G.P. Guedes ${ }^{23}$, E. Guido ${ }^{52,63}$, S. $\mathrm{Hahn}^{41,8}$, P. $\mathrm{Hamal}^{32}$, M.R. Hampel ${ }^{8}$, P. Hansen ${ }^{4}$, D. Harari ${ }^{1}$, V.M. Harvey ${ }^{13}$, A. Haungs ${ }^{41}$, T. Hebbeker ${ }^{42}$, D. Heck ${ }^{41}$, G.C. Hill ${ }^{13}$, C. Hojvat ${ }^{a}$, J.R. Hörandel ${ }^{80,81}$, P. Horvath ${ }^{33}$, M. Hrabovský ${ }^{33}$, T. Huege ${ }^{41,15}$, A. Insolia ${ }^{58,47}$, P.G. Isar $^{74}$, P. Janecek ${ }^{32}$, J.A. Johnsen ${ }^{86}$, J. Jurysek ${ }^{32}$, A. Kääpä ${ }^{38}$, K.H. Kampert ${ }^{38}$, N. Karastathis ${ }^{41}$, B. Keilhauer ${ }^{41}$, J. Kemp ${ }^{42}$, A. Khakurdikar ${ }^{80}$, V.V. Kizakke Covilakam $^{8,41}$, H.O. Klages ${ }^{41}$, M. Kleifges ${ }^{40}$, J. Kleinfeller ${ }^{10}$, M. Köpke ${ }^{39}$, N. Kunka ${ }^{40}$, B.L. Lago ${ }^{17}$, R.G. Lang ${ }^{20}$, N. Langner ${ }^{42}$, M.A. Leigui de Oliveira ${ }^{24}$, V. Lenok $^{41}$, A. Letessier-Selvon ${ }^{35}$, I. LhenryYvon $^{34}$, D. Lo Presti ${ }^{58,47}$, L. Lopes ${ }^{72}$, R. López ${ }^{64}$, L. Lu ${ }^{94}$, Q. Luce ${ }^{39}$, J.P. Lundquist ${ }^{76}$, A. Machado Payeras $^{22}$, G. Mancarella ${ }^{56,48}$, D. Mandat ${ }^{32}$, B.C. Manning ${ }^{13}$, J. Manshanden ${ }^{43}$, P. Mantsch ${ }^{a}$, S. Marafico ${ }^{34}$, A.G. Mariazzi ${ }^{4}$, I.C. Mariş ${ }^{14}$, G. Marsella ${ }^{61,47}$, D. Martello ${ }^{56,48}$, S. Martinelli ${ }^{41,8}$, O. Martínez Bravo ${ }^{64}$, M. Mastrodicasa ${ }^{57,46}$, H.J. Mathes ${ }^{41}$, J. Matthews ${ }^{88}$, G. Matthiae ${ }^{62,51}$, E. Mayotte ${ }^{38}$, P.O. Mazur ${ }^{a}$, G. MedinaTanco $^{68}$, D. Melo ${ }^{8}$, A. Menshikov ${ }^{40}$, K.-D. Merenda ${ }^{86}$, S. Michal ${ }^{33}$, M.I. Micheletti ${ }^{6}$, L. Miramonti ${ }^{59,49}$, S. Mollerach ${ }^{1}$, F. Montanet ${ }^{36}$, C. Morello ${ }^{54,52}$, M. Mostafá ${ }^{91}$, A.L. Müller ${ }^{8}$, M.A. Muller ${ }^{22}$, K. Mulrey ${ }^{15}$, R. Mussa ${ }^{52}$, M. Muzio ${ }^{90}$, W.M. Namasaka ${ }^{38}$, A. Nasr-Esfahani ${ }^{38}$, L. Nellen ${ }^{68}$, M. Niculescu-Oglinzanu ${ }^{73}$, M. Niechciol ${ }^{44}$, D. Nitz ${ }^{89}$, D. Nosek ${ }^{31}$, V. Novotny ${ }^{31}$, L. Nožka ${ }^{33}$, A Nucita ${ }^{56,48}$, L.A. Núñez ${ }^{30}$, M. Palatka ${ }^{32}$, J. Pallotta ${ }^{2}$, P. Papenbreer ${ }^{38}$, G. Parente ${ }^{79}$, A. Parra ${ }^{64}$, J. Pawlowsky ${ }^{38}$, M. Pech ${ }^{32}$, F. Pedreira ${ }^{79}$, J. Pȩkala ${ }^{70}$, R. Pelayo ${ }^{65}$, J. Peña-Rodriguez ${ }^{30}$, E.E. Pereira Martins ${ }^{39,8}$, J. Perez Armand ${ }^{21}$, C. Pérez Bertolli $^{8,41}$, M. Perlin ${ }^{8,41}$, L. Perrone ${ }^{56,48}$, S. Petrera ${ }^{45,46}$, T. Pierog ${ }^{41}$, M. Pimenta ${ }^{72}$, V. Pirronello ${ }^{58,47}$, M. Platino ${ }^{8}$, B. Pont $^{80}$, M. Pothast ${ }^{81,80}$, P. Privitera ${ }^{92}$, M. Prouza ${ }^{32}$, A. Puyleart ${ }^{89}$, S. Querchfeld ${ }^{38}$, J. Rautenberg ${ }^{38}$, D. Ravignani ${ }^{8}$, M. Reininghaus ${ }^{41,8}$, J. Ridky ${ }^{32}$, F. Riehn ${ }^{72}$, M. Risse ${ }^{44}$, V. Rizi ${ }^{57,46}$, W. Rodrigues de Carvalho ${ }^{21}$, J. Rodriguez Rojo ${ }^{11}$, M.J. Roncoroni ${ }^{8}$, S. Rossoni ${ }^{43}$, M. Roth ${ }^{41}$, E. Roulet ${ }^{1}$, A.C. Rovero ${ }^{5}$, P. Ruehl ${ }^{44}$, A. Saftoiu ${ }^{73}$, F. Salamida ${ }^{57,46}$, H. Salazar ${ }^{64}$, G. Salina ${ }^{51}$, J.D. Sanabria Gomez ${ }^{30}$, F. Sánchez ${ }^{8}$, E.M. Santos ${ }^{21}$, E. Santos ${ }^{32}$, F. Sarazin ${ }^{86}$, R. Sarmento ${ }^{72}$, C. Sarmiento-Cano ${ }^{8}$, R. Sato ${ }^{11}$, 
P. Savina ${ }^{56,48,34,94}$, C.M. Schäfer ${ }^{41}$, V. Scherini ${ }^{56,48}$, H. Schieler ${ }^{41}$, M. Schimassek ${ }^{39,8}$, M. Schimp ${ }^{38}$, F. Schlüter ${ }^{41,8}$, D. Schmidt ${ }^{39}$, O. Scholten ${ }^{84,15}$, P. Schovánek ${ }^{32}$, F.G. Schröder ${ }^{93,41}$, S. Schröder ${ }^{38}$, J. Schulte ${ }^{42}$, S.J. Sciutto ${ }^{4}$, M. Scornavacche ${ }^{8,41}$, A. Segreto ${ }^{53,47}$, S. Sehgal ${ }^{38}$, R.C. Shellard ${ }^{16}$, G. Sigl ${ }^{43}$, G. Silli ${ }^{8,41}$, O. Sima ${ }^{73, f}$, R. Šmída ${ }^{92}$, P. Sommers ${ }^{91}$, J.F. Soriano ${ }^{87}$, J. Souchard ${ }^{36}$, R. Squartini ${ }^{10}$, M. Stadelmaier ${ }^{41,8}$, D. Stanca ${ }^{73}$, S. Stanič ${ }^{76}$, J. Stasielak ${ }^{70}$, P. Stassi ${ }^{36}$, A. Streich ${ }^{39,8}$, M. Suárez-Durán ${ }^{14}$, T. Sudholz ${ }^{13}$, T. Suomijärvi ${ }^{37}$, A.D. Supanitsky ${ }^{8}$, Z. Szadkowski ${ }^{71}$, A. Tapia ${ }^{29}$, C. Taricco ${ }^{63,52}$, C. Timmermans ${ }^{81,80}$, O. Tkachenko ${ }^{41}$, P. Tobiska ${ }^{32}$, C.J. Todero Peixoto ${ }^{19}$, B. Tomé ${ }^{72}$, Z. Torrès ${ }^{36}$, A. Travaini ${ }^{10}$, P. Travnicek $^{32}$, C. Trimarelli ${ }^{57,46}$, M. Tueros ${ }^{4}$, R. Ulrich ${ }^{41}$, M. Unger ${ }^{41}$, L. Vaclavek ${ }^{33}$, M. Vacula ${ }^{33}$, J.F. Valdés Galicia ${ }^{68}$, L. Valore ${ }^{60,50}$, E. Varela ${ }^{64}$, A. Vásquez-Ramírez ${ }^{30}$, D. Veberič ${ }^{41}$, C. Ventura ${ }^{27}$, I.D. Vergara Quispe ${ }^{4}$, V. Verzi ${ }^{51}$, J. Vicha ${ }^{32}$, J. Vink $^{83}$, S. Vorobiov ${ }^{76}$, H. Wahlberg ${ }^{4}$, C. Watanabe ${ }^{26}$, A.A. Watson ${ }^{c}$, M. Weber ${ }^{40}$, A. Weindl ${ }^{41}$, L. Wiencke ${ }^{86}$, H. Wilczyński ${ }^{70}$, M. Wirtz ${ }^{42}$, D. Wittkowski ${ }^{38}$, B. Wundheiler ${ }^{8}$, A. Yushkov $^{32}$, O. Zapparrata ${ }^{14}$, E. Zas ${ }^{79}$, D. Zavrtanik ${ }^{76,77}$, M. Zavrtanik ${ }^{77,76}$, L. Zehrer ${ }^{76}$

${ }^{1}$ Centro Atómico Bariloche and Instituto Balseiro (CNEA-UNCuyo-CONICET), San Carlos de Bariloche, Argentina

${ }^{2}$ Centro de Investigaciones en Láseres y Aplicaciones, CITEDEF and CONICET, Villa Martelli, Argentina

${ }^{3}$ Departamento de Física and Departamento de Ciencias de la Atmósfera y los Océanos, FCEyN, Universidad de Buenos Aires and CONICET, Buenos Aires, Argentina

${ }^{4}$ IFLP, Universidad Nacional de La Plata and CONICET, La Plata, Argentina

${ }^{5}$ Instituto de Astronomía y Física del Espacio (IAFE, CONICET-UBA), Buenos Aires, Argentina

${ }^{6}$ Instituto de Física de Rosario (IFIR) - CONICET/U.N.R. and Facultad de Ciencias Bioquímicas y Farmacéuticas U.N.R., Rosario, Argentina

${ }^{7}$ Instituto de Tecnologías en Detección y Astropartículas (CNEA, CONICET, UNSAM), and Universidad Tecnológica Nacional - Facultad Regional Mendoza (CONICET/CNEA), Mendoza, Argentina

${ }^{8}$ Instituto de Tecnologías en Detección y Astropartículas (CNEA, CONICET, UNSAM), Buenos Aires, Argentina

${ }^{9}$ International Center of Advanced Studies and Instituto de Ciencias Físicas, ECyT-UNSAM and CONICET, Campus Miguelete - San Martín, Buenos Aires, Argentina

${ }^{10}$ Observatorio Pierre Auger, Malargüe, Argentina

${ }^{11}$ Observatorio Pierre Auger and Comisión Nacional de Energía Atómica, Malargüe, Argentina

12 Universidad Tecnológica Nacional - Facultad Regional Buenos Aires, Buenos Aires, Argentina

${ }^{13}$ University of Adelaide, Adelaide, S.A., Australia

14 Université Libre de Bruxelles (ULB), Brussels, Belgium

15 Vrije Universiteit Brussels, Brussels, Belgium

${ }^{16}$ Centro Brasileiro de Pesquisas Fisicas, Rio de Janeiro, RJ, Brazil

${ }^{17}$ Centro Federal de Educação Tecnológica Celso Suckow da Fonseca, Nova Friburgo, Brazil

${ }^{18}$ Instituto Federal de Educação, Ciência e Tecnologia do Rio de Janeiro (IFRJ), Brazil

${ }^{19}$ Universidade de São Paulo, Escola de Engenharia de Lorena, Lorena, SP, Brazil

${ }^{20}$ Universidade de São Paulo, Instituto de Física de São Carlos, São Carlos, SP, Brazil

${ }^{21}$ Universidade de São Paulo, Instituto de Física, São Paulo, SP, Brazil

22 Universidade Estadual de Campinas, IFGW, Campinas, SP, Brazil

${ }^{23}$ Universidade Estadual de Feira de Santana, Feira de Santana, Brazil

${ }^{24}$ Universidade Federal do ABC, Santo André, SP, Brazil

${ }^{25}$ Universidade Federal do Paraná, Setor Palotina, Palotina, Brazil

${ }^{26}$ Universidade Federal do Rio de Janeiro, Instituto de Física, Rio de Janeiro, RJ, Brazil

${ }^{27}$ Universidade Federal do Rio de Janeiro (UFRJ), Observatório do Valongo, Rio de Janeiro, RJ, Brazil

${ }^{28}$ Universidade Federal Fluminense, EEIMVR, Volta Redonda, RJ, Brazil

${ }^{29}$ Universidad de Medellín, Medellín, Colombia

${ }^{30}$ Universidad Industrial de Santander, Bucaramanga, Colombia

${ }^{31}$ Charles University, Faculty of Mathematics and Physics, Institute of Particle and Nuclear Physics, Prague, Czech Republic

32 Institute of Physics of the Czech Academy of Sciences, Prague, Czech Republic 
${ }^{33}$ Palacky University, RCPTM, Olomouc, Czech Republic

34 CNRS/IN2P3, IJCLab, Université Paris-Saclay, Orsay, France

${ }^{35}$ Laboratoire de Physique Nucléaire et de Hautes Energies (LPNHE), Sorbonne Université, Université de Paris, CNRSIN2P3, Paris, France

${ }^{36}$ Univ. Grenoble Alpes, CNRS, Grenoble Institute of Engineering Univ. Grenoble Alpes, LPSC-IN2P3, 38000 Grenoble, France

${ }^{37}$ Université Paris-Saclay, CNRS/IN2P3, IJCLab, Orsay, France

38 Bergische Universität Wuppertal, Department of Physics, Wuppertal, Germany

${ }^{39}$ Karlsruhe Institute of Technology (KIT), Institute for Experimental Particle Physics, Karlsruhe, Germany

${ }^{40}$ Karlsruhe Institute of Technology (KIT), Institut für Prozessdatenverarbeitung und Elektronik, Karlsruhe, Germany

${ }^{41}$ Karlsruhe Institute of Technology (KIT), Institute for Astroparticle Physics, Karlsruhe, Germany

${ }^{42}$ RWTH Aachen University, III. Physikalisches Institut A, Aachen, Germany

${ }^{43}$ Universität Hamburg, II. Institut für Theoretische Physik, Hamburg, Germany

44 Universität Siegen, Department Physik - Experimentelle Teilchenphysik, Siegen, Germany

${ }^{45}$ Gran Sasso Science Institute, L'Aquila, Italy

46 INFN Laboratori Nazionali del Gran Sasso, Assergi (L'Aquila), Italy

${ }^{47}$ INFN, Sezione di Catania, Catania, Italy

${ }^{48}$ INFN, Sezione di Lecce, Lecce, Italy

${ }^{49}$ INFN, Sezione di Milano, Milano, Italy

${ }^{50}$ INFN, Sezione di Napoli, Napoli, Italy

${ }^{51}$ INFN, Sezione di Roma "Tor Vergata", Roma, Italy

52 INFN, Sezione di Torino, Torino, Italy

53 Istituto di Astrofisica Spaziale e Fisica Cosmica di Palermo (INAF), Palermo, Italy

54 Osservatorio Astrofisico di Torino (INAF), Torino, Italy

55 Politecnico di Milano, Dipartimento di Scienze e Tecnologie Aerospaziali , Milano, Italy

56 Università del Salento, Dipartimento di Matematica e Fisica "E. De Giorgi”, Lecce, Italy

57 Università dell'Aquila, Dipartimento di Scienze Fisiche e Chimiche, L'Aquila, Italy

58 Università di Catania, Dipartimento di Fisica e Astronomia, Catania, Italy

${ }^{59}$ Università di Milano, Dipartimento di Fisica, Milano, Italy

${ }^{60}$ Università di Napoli "Federico II", Dipartimento di Fisica "Ettore Pancini”, Napoli, Italy

${ }^{61}$ Università di Palermo, Dipartimento di Fisica e Chimica "E. Segrè", Palermo, Italy

62 Università di Roma “Tor Vergata”, Dipartimento di Fisica, Roma, Italy

${ }^{63}$ Università Torino, Dipartimento di Fisica, Torino, Italy

64 Benemérita Universidad Autónoma de Puebla, Puebla, México

${ }^{65}$ Unidad Profesional Interdisciplinaria en Ingeniería y Tecnologías Avanzadas del Instituto Politécnico Nacional (UPIITA-IPN), México, D.F., México

66 Universidad Autónoma de Chiapas, Tuxtla Gutiérrez, Chiapas, México

${ }^{67}$ Universidad Michoacana de San Nicolás de Hidalgo, Morelia, Michoacán, México

${ }^{68}$ Universidad Nacional Autónoma de México, México, D.F., México

${ }^{69}$ Universidad Nacional de San Agustin de Arequipa, Facultad de Ciencias Naturales y Formales, Arequipa, Peru

${ }^{70}$ Institute of Nuclear Physics PAN, Krakow, Poland

${ }^{71}$ University of Łódź, Faculty of High-Energy Astrophysics,Lódź, Poland

${ }^{72}$ Laboratório de Instrumentação e Física Experimental de Partículas - LIP and Instituto Superior Técnico - IST, Universidade de Lisboa - UL, Lisboa, Portugal

73 "Horia Hulubei” National Institute for Physics and Nuclear Engineering, Bucharest-Magurele, Romania

${ }^{74}$ Institute of Space Science, Bucharest-Magurele, Romania

75 University Politehnica of Bucharest, Bucharest, Romania

76 Center for Astrophysics and Cosmology (CAC), University of Nova Gorica, Nova Gorica, Slovenia

${ }^{77}$ Experimental Particle Physics Department, J. Stefan Institute, Ljubljana, Slovenia

78 Universidad de Granada and C.A.F.P.E., Granada, Spain

${ }^{79}$ Instituto Galego de Física de Altas Enerxías (IGFAE), Universidade de Santiago de Compostela, Santiago de Compostela, Spain 
${ }^{80}$ IMAPP, Radboud University Nijmegen, Nijmegen, The Netherlands

${ }^{81}$ Nationaal Instituut voor Kernfysica en Hoge Energie Fysica (NIKHEF), Science Park, Amsterdam, The Netherlands

82 Stichting Astronomisch Onderzoek in Nederland (ASTRON), Dwingeloo, The Netherlands

${ }^{83}$ Universiteit van Amsterdam, Faculty of Science, Amsterdam, The Netherlands

${ }^{84}$ University of Groningen, Kapteyn Astronomical Institute, Groningen, The Netherlands

85 Case Western Reserve University, Cleveland, OH, USA

86 Colorado School of Mines, Golden, CO, USA

${ }^{87}$ Department of Physics and Astronomy, Lehman College, City University of New York, Bronx, NY, USA

${ }^{88}$ Louisiana State University, Baton Rouge, LA, USA

${ }^{89}$ Michigan Technological University, Houghton, MI, USA

${ }^{90}$ New York University, New York, NY, USA

${ }^{91}$ Pennsylvania State University, University Park, PA, USA

92 University of Chicago, Enrico Fermi Institute, Chicago, IL, USA

93 University of Delaware, Department of Physics and Astronomy, Bartol Research Institute, Newark, DE, USA

94 University of Wisconsin-Madison, Department of Physics and WIPAC, Madison, WI, USA

${ }^{a}$ Fermi National Accelerator Laboratory, Fermilab, Batavia, IL, USA

${ }^{b}$ Max-Planck-Institut für Radioastronomie, Bonn, Germany

${ }^{c}$ School of Physics and Astronomy, University of Leeds, Leeds, United Kingdom

${ }^{d}$ Colorado State University, Fort Collins, CO, USA

$e^{e}$ now at Hakubi Center for Advanced Research and Graduate School of Science, Kyoto University, Kyoto, Japan

$f$ also at University of Bucharest, Physics Department, Bucharest, Romania 